\title{
How to ensure clitoral bud survival in a sexual reassignment surgery for transsexualism
}

\author{
Juthapot Pumsup, MD \\ Juthapot Clinics, Trad, Thailand
}

Background: Sexual reassignment surgery (SRS) is the complicated procedure as it has a very high risk of complications. The loss of clitoris is the ones. Accordingly, surgeons should carefully consider the surgical technique and ensure no mistakes during operation. Although most surgeons perform the operation carefully, a considerable incidence of clitoral bud necrosis has been reported. Thus, finding techniques that improve the success rates in surgeries is very important.

Objective: We aimed to study the cause of neo-clitoral bud necrosis after SRS in order to devise a mechanism to avoid neo-clitoral bud necrosis, and to find a surgical technique for ensuring the survival of the clitoral bud.

Methods: The study was conducted in 20 patients, who underwent a male-to-female SRS via Author technique From Juthapot Clinics, Trad Hospital, and Private Hospital (couldn't mention) during September 2016 to August 2017. This intervention included various factors as mention below.

Results: Of the 20 patients who underwent the procedure with this technique, 18 patients were without clitoral bud necrosis and 2 patients had partial clitoral bud necrosis at the tip. Sensation was preserved in these patients, although it was decreased. The sensation has 2 part: the 1st part is neoclitoris and the 2 nd is at the anterior vagina, that made by the urethral lining after spatulation, that can serve the sensation.

Conclusion: This technique may be useful for increasing the survival rate of clitoral buds. Further, this technique comprised multiple factors to ensure the success of the procedure. We found that sharp-scissor dissection promoted better outcomes than electrocautery dissection because of the thermal effect. Moreover, bipolar electrocautery procedures should be used to stop bleeding. Dissection should be deep to remove some layers of tunica albuginea. Furthermore, the width of the neurovascular strip must cover the dorsal nerve laterally. The clitoral bud size was not related to necrosis, but was associated with sensation. Finally, one must be careful about neurovascular strip compression, kinking, and twisting to ensure surgical success.

Keywords: clitoris; clitoral bud; clitoral bud survive; neo-clitoris; sexual reassignment surgery; transgender surgery

\section{Introduction}

Sexual reassignment surgery (SRS) is the complicated procedure as it has a very high risk of complications. One of the complications of the male-to-female transsexual surgery is the loss of clitoris. Accordingly, surgeons should carefully consider the surgical technique and ensure no mistakes during operation. Although most surgeons perform the operation carefully, a considerable incidence of clitoral bud necrosis has been reported. Thus, finding techniques that improve the success rates in surgeries is very important for achieving the purpose of the surgery.

Received January 23, 2018; Revised June 12, 2018; Accepted June 14, 2018

Corresponding author: Juthapot Pumsup

E-mail: ship1md@yahoo.com, ORCID: https://orcid.org/0000-0001-7355-2154

This is an Open Access article distributed under the terms of the Creative Commons Attribution Non-Commercial License (http://creativecommons.org/licenses/by-nc/4.0), which permits unrestricted non-commercial use, distribution, and reproduction in any medium, provided the original work is properly cited.

Copyright @ 2018 Korean Society of Korean Cosmetic Surgery and Medicine (KSKCS \& KCCS). 


\section{Basic knowledge of the penis and its neurovascular supplies}

Three columnar bodies are attached to be the penis, and a pair of dorsally columnar bodies extend along the penis (Fig. 1) [1]. They contain the cavernosal vascular tissue called the corpus cavernosum. Proximally, the corpus cavernosum tapers to form the crus, which is attached to the pubic arch. The two corporal bodies are surrounded by a strong $1-\mathrm{mm}$ thick fascial sheath called tunica albuginea, which forms a septum called septum pectiniforme (Pectiniform septa) to separate each corpora. The ischiocavernosus muscles arise from the inner surface of the ischial tuberosity to support the corpus cavernosum, and are innervated by the perineal nerves. The third columnar body of the penis is the corpus spongiosum, which contains the urethra, and its distal end is the glans of the penis. The corpus spongiosum is attached proximally to the perineal membrane and the most proximal portion is larger, forming the bulb (bulbous part of urethra). Buck's fascia is the surrounding fascia of the three corporal bodies, which is a continuation of Colles' fascia. The Buck's fascia is attached posteriorly to the urogenital diaphragm, and it anteriorly forms the suspensory ligament. There are intercommunicating vascular vessels between the two corpus cavernosa passing via pectiniform septa in the distal corpora.

\section{Arterial supply}

The superficial penile artery (dorsal artery of the penis) lies between superficial penile fascia and Buck's fascia (Fig. 1) [1]. It originates from the external pudendal artery, which is a branch of the femoral artery and corresponding vein to supply to the penile skin and is located between the superficial penile fascia and the Buck's fascia. The deep arterial supply (arteria profunda penis) arises from the internal pudendal artery [2-4], which is a branch of the internal iliac artery and then branches into the penile artery. The penile artery exits the urogenital diaphragm into three branches, the bulbourethral, cavernous, and urethral arteries. The cavernous artery directly supplies the corpora cavernosa. The cavernous artery continues along the corpora cavernosa as the deep dorsal artery. The corpus spongiosum is supplied by the bulbourethral artery (arteria bulbi penis) proximally, and by the circumflex arteries, which branch from the dorsal artery, along its shaft. The glans of the penis is supplied by the deep dorsal artery. The deep dorsal artery, the deep dorsal vein, and the dorsal nerve are enclosed within the Buck's fascia.

\section{Venous drainage}

The superficial penile vein drains into the external pudendal vein. The circumflex and deep dorsal veins drain into Santorini's plexus (prostatic venous plexus), as do the crural and cavernosal veins [2-4].

\section{Nerve innervation}

The two spinal autonomic centers are as follows: The parasympathetic spinal center is located at levels S2 to S4 and the sympathetic spinal center is located at levels T12 to L2 form the inferior hypogastric plexus, and these send nerve fibers to the pelvic organs [5-7].

\section{The cavernous nerves of the penis}

They are autonomic nerve fiber branches of the inferior hypogastric plexus, located posterolaterally to the seminal vesicles that pass laterally to the prostate with the main location at 5 and 7 o'clock directions. When they pass toward the membranous
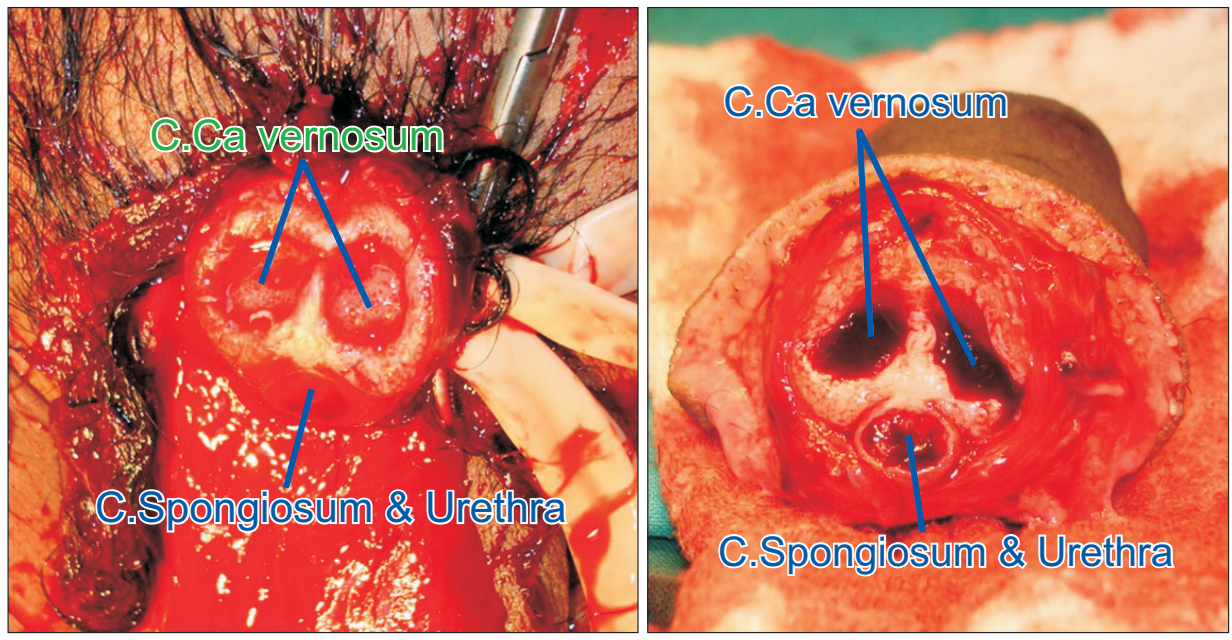

Fig. 1. Amputation of the penis and anatomy. 
urethra, the fibers are located at 3 and 9 o'clock directions and at the distal bulb of the penis, they are located at 1 and 11 O'clock directions, where they enter the penis. In addition, nerve fibers accompany the arteries or sensory nerves. The autonomic nerve fibers innervate the helicine arteries. The cholinergic nerve endings stimulate nitric oxide (NO)-synthase and release NO. This is the physiologic mechanism underlying erection must have the vasodilation.

\section{Motor innervation}

The pudendal nerve (S2-S4) innervates the bulbocavernosus muscle and ischiocavernosus muscle.

\section{Sensory innervation}

The dorsal penile nerve and pudendal nerve send impulses to the receptor of the afferent nerve fibers in the spinal cord, which lead toward either the medial lemniscus or spinothalamic tract.

\section{Aims}

1. To study the cause of neo-clitoral bud necrosis after SRS surgery.

2. To device a mechanism to avoid neo-clitoral bud necrosis during the SRS surgery.

3. To find a successful technique for ensuring the survival of the clitoral bud.

\section{Materials and methods}

The study was conducted in 20 patients, who underwent a male-to-female SRS with Author technique for ensuring the survival of the clitoral bud from Juthapot Clinics, Trad Hospital, and Private Hospital (couldn't mention) during September 2016 to August 2017. This intervention included various factors such as deep dorsal neurovascular bundle dissection, appropriate use of equipment, technique for ensuring appropriate depth and width of the dorsal neurovascular strip, accurate technique for clitoral bud placement and techniques for avoiding neurovascular strip compression, kinking, and twisting.

\section{Procedures}

The procedures leading to surgical success with respect to survival of the clitoral bud and preservation of clitoral bud sensation were multifactorial including deep dorsal neurovascular bundle dissection technique, appropriate use of equipment, and accurate techniques, and clitoral bud placement.

\section{Dissection}

Some part of the glans of the penis and coronal sulcus comprise the neo-clitoral bud, and necrosis could occur here if the surgeons did not keep in mind the deep dorsal neurovascular bundles during dissected. The deep dorsal neurovascular bundle lies under the Buck's fascia and over the tunica albuginea, which was the plane of dissection. During dissection, a deep cut was made to remove some tissue layers of tunica albuginea, but this should be carefully visualized with an attempt to cause minimal vascular trauma. A sharp dissection with sharp scissors was found to be safer than other techniques. Bleeding from the circumflex vascular branch was stopped by a minor coagulated electrocautery procedure, in particular, the bipolar cautery procedure, as it was minor vascular trauma [8]. The patient should be asked for the preference with regard to keeping corpus cavernosum or the surgeon's preference for cavernosum sparing should be considered. If cavernosum is not to be preserved, the procedure might require a deeper cut in order to dissect all layers of the tunica albuginea, and took the deep dorsal neurovascular bundle. This was advantageous to decrease bleeding from the emissary vein and the circumflex vascular branch. We made the dissection at the corpora along the penile length until the pubic arch. While performing this dissection, one must be careful at 1 and 11 o'clock directions because these are the points where the vascular vessels and nerves are located.

\section{Electrocautery dissection}

Dissections using electrocautery procedures should be avoided because the thermal effect could promote microvascular injuries. However, the decision should be made depending on the surgeon's hand skills. Some surgeons prefer using electrocautery procedures to make a wide strip cut and remove all layers of tunica albuginea and Buck's fascia successfully. One should be aware of thermal injuries in thicker tissue, which occur easily occur in thicker tissues than in thinner tissues, and of the fact that heat is released slowly in the thicker layers.

\section{The depth of the dissection}

The dissection was made by piercing some layers of tunica albuginea [9], and the emissary veins could be seen bleeding (Fig. 2). We preferred to stop the bleeding by the bipolar diathermy procedure to preserve the nerve fibers inside the bundle. Other surgeons prefer ligation with $4 / 0$ or $5 / 0$ silk ties. The dissection should be as deep as possible (Fig. 3) to avoid a possible vascular injury. 


\section{The width of the dissection}

The deep dorsal neurovascular bundle comprises the following: 1) The deep dorsal nerve, which runs lateral to the penis; 2) The deep dorsal artery, which runs medial to the penis; and 3) The deep dorsal vein, which runs through the midline of the penis.

The incision was made as wide as possible as we could not clearly identify the nerve fibers. The guideline to make the incision was about 2 to 3 o'clock on the left of the patient and 9 to 10 o'clock on the right of the patient. This incision was safer for the nerve fiber. The incision was parallel, and along the penile shaft, and depth was until some layers of tunica albuginea.

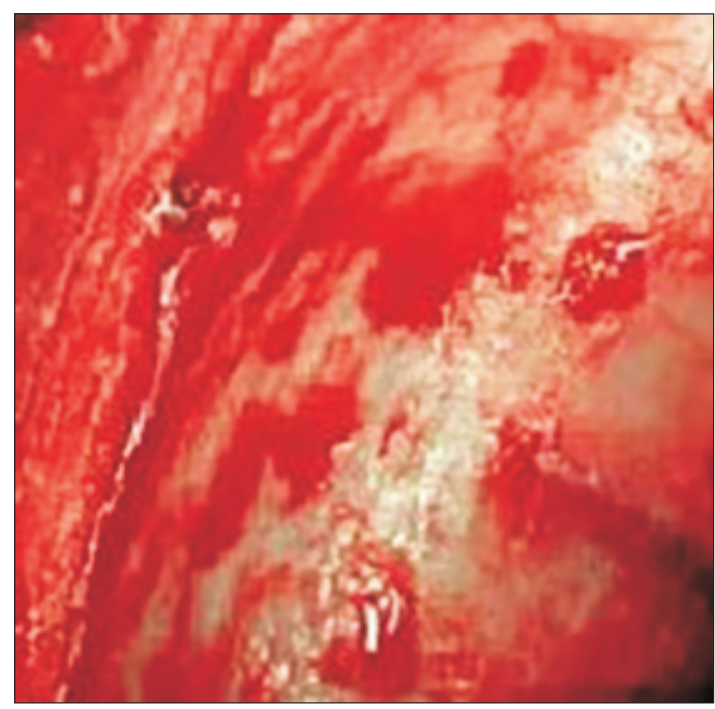

Fig. 2. The depth of the dissection and the bleeding emissary vein end.

\section{Clitoral bud size}

The size of clitoral bud was not related to the increasing risk of clitoral bud necrosis, but partial necrosis was observed in buds with a high internal pressure after the bud was sutured. We observed epithelial white scales on the clitoral bud with shading. However, the clitoral bud would survive even after complete shading. The sign of necrosis is a progressive black color of the clitoral bud. This is an irreversible presentation.

\section{Neurovascular strip compression, kinking, and twist}

To avoid clitoral bud necrosis one should re-check the neurovascular strip compression, kinking, and twist. The strip must be free, with no strip compression, twist, or kinking.

The cause of the neurovascular strip compression, kinking, and twist were as follows:

1. Vaginal tube placement: when a long strip was attached to the subcutaneous tissue of the vaginal tube during vaginal tube invagination, it was pulled down to the inferior pubic rami bone above the anterior neovaginal wall. The strip was checked and made free for ensuring survival at the left or right side of the vaginal tube and the space free. The strip fixed to the soft tissue was unnecessary.

2. The strip was checked for kinking or twisting while placing the clitoral bud at the proper location. It should not kink or twist.

3. The hole of clitoral bud deliver could be made with two techniques.

a. Penile skin hole include Dartos fascia: Dartos hole was very tight. However, we took risk of compression with tightening. The hole should be wide enough in order to prevent necrosis.
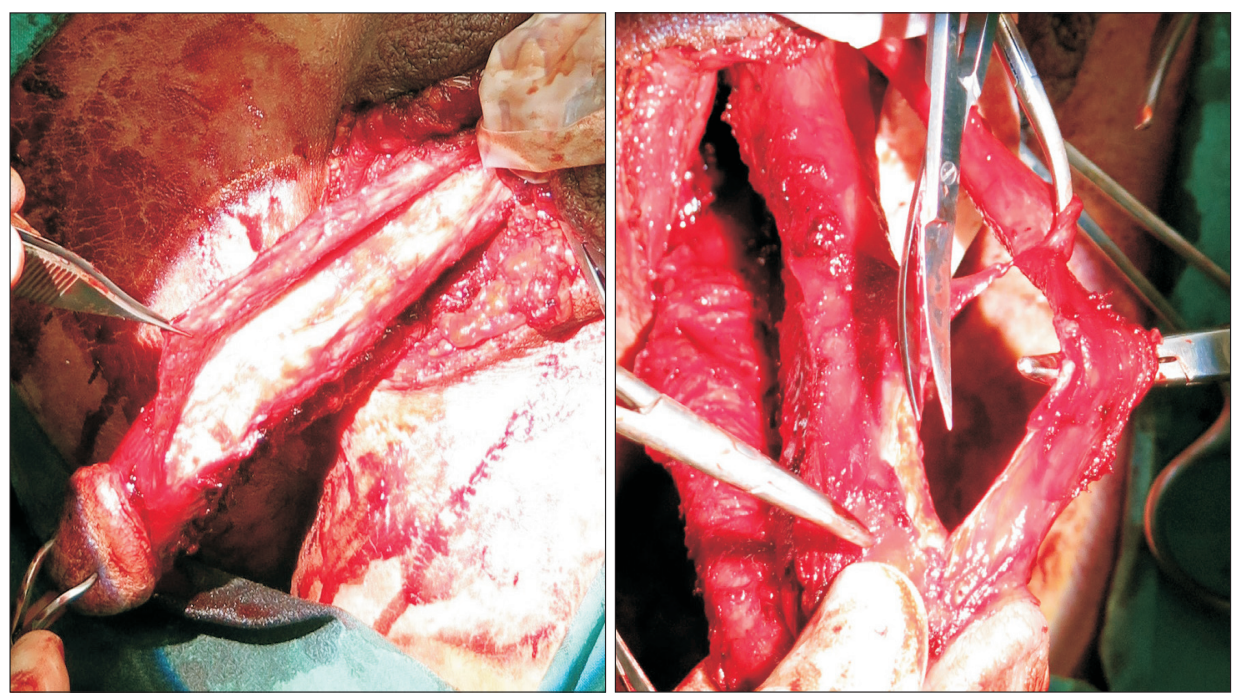

Fig. 3. Deep dorsal neurovascular dissection close to tunica albuginea. 
b. Dartos hole: after the penoscrotal skin was separated, the hole made with the sharp scissors penetrated to the dartos tissue longitudinally. This helped decreasing the risk of the clitoral bud compression and increasing the neovaginal depth.

4. Postoperative vaginal packing pressure: The packing should be tight inside, but the introitus must be loose. Vaginal packing is used for decreased internal pelvic bleeding and stabilizing the neovaginal tube. Vaginal packing should not stop bleeding in the introitus. Unnecessary to tight packing at the introitus.

5. Accidental suture ligation during the labiaplasty: The surgeon must remember the side of the strip first. Further, the depth of suture proper should not be too deep. Surgeon should open the labial flap and see the suture stitch by stitch.

6. Clitoral bud and strip angulation: The clitoral bud was placed and if the clitoral bud was in the high position or anterior to the inferior pubic rami and the clitoral bud tip downward to introitus, labiaplasty was performed. When the skin was stretched to create the labia, the force could have been increased while compression, increasing the angulation. This may have caused the clitoral blood supply to cut, and led to clitoral bud necrosis.

\section{Results}

The surgeries in 20 patients were performed with the abovementioned technique. In all, 18 patients did not report clitoral bud necrosis, and 2 patients had partial clitoral bud necrosis at the tip. The sensation was still presented, although the sensation was decreased. However, no patient complained as the second sensation from the introitus and anterior vagina was preserved by the spatulated urethral lining.

\section{Conclusion}

Neurovascular dissection was the delicate procedure during SRS. Clitoral bud survival is of utmost importance in most transgender surgeries. The external genital shape and look are secondary. During sexual intercourse, the sensation of the clitoral bud is important for the female and her partner. The technique reported here may be useful for increasing the clitoral bud survival rate. The technique comprises multiple factors for the success of the procedure. A sharp scissor dissection could promote a better outcome than electrocautery dissection because of the thermal effect. Further, bipolar electrocautery procedure should be used to stop bleeding. Dissection should be deep to remove some layers of tunica albuginea. Moreover, the width of the neurovascular strip must cover the dorsal nerve laterally. The clitoral bud size was not related to necrosis, but partial necrosis was seen in buds with high internal pressure after the bud was sutured. The size of the clitoral bud was associated with the sensation. The sensation in the bigger buds was better than that in the smaller ones. Finally, surgeon must be careful about neurovascular strip compression, kinking, and twisting to ensure surgical success.

\section{Discussion}

Neurovascular dissection was the delicate reconstructive procedure. The loss of neo-clitoral bud in male-to-female SRS was the one of the serious problems. A rate of $1 \%$ for neo-clitoral bud necrosis in the patients with SRS may be high. The outcome of neo-clitoral survival can be achieved by multiple techniques as describe above. Trying to find such new techniques can decrease the risk of neo-clitoral bud necrosis. However, these techniques are not widely known and many surgeons do not believe in sharing their successful experiences, especially in Thailand. The technique described here was give-and-take in a special group. This study showed the success of operation with regard to the survival of the clitoral bud as all 20 cases of maleto-female SRS showed survival of the clitoris. Although partial necrosis was observed in 2 cases at the tip of bud, sensation was still preserved.

\section{Conflicts of interest}

The author has nothing to disclose.

\section{References}

1. Netter FH. Perineum and external genitalia: male. In: Netter FH, editor. Atlas of human anatomy. 6th ed. Philadelphia: Saunders/Elsevier; 2014. p. 359-61.

2. Netter FH. Arteries and veins of pelvis: male. In: Netter FH, editor. Atlas of human anatomy. 6th ed. Philadelphia, PA: Saunders/Elsevier; 2014. p. 381, 383.

3. Rohen JW, Yokochi C, Lütjen-Drecoll E. Male external genital organs. In: Rohen JW, Yokochi C, Lütjen-Drecoll E, editors. Color atlas of anatomy: a photographic study of the human body. 7th ed. Philadelphia, PA: Wolters Kluwer, Lippincott Williams \& Wilkins; 2011. p. 340. 
4. Rohen JW, Yokochi C, Lütjen-Drecoll E. Pelvic cavity in male: vessels of pelvic organs. In: Rohen JW, Yokochi C, LütjenDrecoll E, editors. Color atlas of anatomy: a photographic study of the human body. 7th ed. Philadelphia, PA: Wolters Kluwer, Lippincott Williams \& Wilkins; 2011.p. 346-7.

5. Netter FH. Nerve of external genitalia: male. In: Netter FH, editor. Atlas of human anatomy. 6th ed. Philadelphia, PA: Saunders/Elsevier; 2014. p. 387-8.

6. Netter FH. Nerve of perineum: male. In: Netter FH, editor. Atlas of human anatomy. 6th ed. Philadelphia, PA: Saunders/ Elsevier; 2014. p. 389.

7. Netter FH. Innervation of male reproductive organs: schema.
In: Netter FH, editor. Atlas of human anatomy. 6th ed. Philadelphia, PA: Saunders/Elsevier; 2014. p. 394.

8. Hag J. Vaginoplasty in male to female transsexuals by inversion of penile and scrotal skin. In: Ehrlich RM, Alter GJ, editors. Reconstructive and plastic surgery of the external genitalia: adult and pediatric. Philadelphia, PA: Saunders; 1999. p. 294-300.

9. Soli M, Brunocilla E, Bertaccini A, Palmieri F, Barbieri B, Martorana G. Male to female gender reassignment: modified surgical technique for creating the neoclitoris and mons veneris. J Sex Med 2008;5:210-6. 